\title{
Characterization of FN1-FGFR1 and novel FN1-FGF1 fusion genes in a large series of phosphaturic mesenchymal tumors
}

Jen-Chieh Lee ${ }^{1}$, Sheng-Yao Su ${ }^{2,3,21}$, Chun A Changou ${ }^{4,5,21}$, Rong-Sen Yang ${ }^{6}$, Keh-Sung Tsai ${ }^{7}$, Michael T Collins ${ }^{8}$, Eric S Orwoll ${ }^{\text {, Chung-Yen Lin }}{ }^{2}$, Shu-Hwa Chen ${ }^{2}$, Shyang-Rong Shih ${ }^{7}$, Cheng-Han Lee ${ }^{10}$, Yoshinao Oda ${ }^{11}$, Steven D Billings ${ }^{12}$, Chien-Feng Li ${ }^{13}$, G Petur Nielsen ${ }^{14}$, Eiichi Konishi ${ }^{15}$, Fredrik Petersson ${ }^{16}$, Thomas O Carpenter ${ }^{17}$, Kesavan Sittampalam ${ }^{18}$, Hsuan-Ying Huang ${ }^{19}$ and Andrew L Folpe ${ }^{20}$

${ }^{1}$ Department and Graduate Institute of Pathology, National Taiwan University Hospital, National Taiwan University College of Medicine, Taipei, Taiwan; ${ }^{2}$ Institute of Information Science, Academia Sinica, Taipei, Taiwan; ${ }^{3}$ Bioinformatics Program, Taiwan International Graduate Program, Academia Sinica, Institute of Biomedical Informatics, National Yang-Ming University, Taipei, Taiwan; ${ }^{4}$ Graduate Institute of Cancer Biology and Drug Discovery, Taipei Medical University, Taipei, Taiwan; ${ }^{5}$ Integrated Laboratory, Center of Translational Medicine, Graduate Institute of Translational Medicine, Taipei Medical University, Taipei, Taiwan; ${ }^{6}$ Department of Orthopedics, National Taiwan University Hospital, National Taiwan University College of Medicine, Taipei, Taiwan; ${ }^{7}$ Department of Internal Medicine, National Taiwan University Hospital, National Taiwan University College of Medicine, Taipei, Taiwan; ${ }^{8}$ Skeletal Clinical Studies Unit, National Institutes of Health, Bethesda, MD, USA; ${ }^{9}$ Bone and Mineral Unit, Oregon Health and Science University, Portland, OR, USA; ${ }^{10}$ Department of Laboratory Medicine and Pathology, University of Alberta and Royal Alexandra Hospital, Edmonton, Alberta, Canada; ${ }^{11}$ Department of Anatomic Pathology, Graduate School of Medical Sciences, Kyushu University, Fukuoka, Japan; ${ }^{12}$ Department of Pathology, Cleveland Clinic, Cleveland, OH, USA; ${ }^{13}$ Department of Pathology, Chi-Mei Medical Center, Tainan, Taiwan; ${ }^{14}$ Department of Pathology, Massachusetts General Hospital, Boston, MA, USA; ${ }^{15}$ Department of Pathology, Kyoto Prefectural University of Medicine, Kyoto, Japan; ${ }^{16}$ Department of Pathology, National University Health System, Singapore, Singapore; ${ }^{17}$ Department of Pediatrics (Endocrinology), Yale University School of Medicine, New Haven, CT, USA; ${ }^{18}$ Pathology Department, Singapore General Hospital, Singapore, Singapore; ${ }^{19}$ Department of Pathology, Kaohsiung Chang Gung Memorial Hospital, Chang Gung University College of Medicine, Kaohsiung, Taiwan and ${ }^{20}$ Department of Laboratory Medicine and Pathology, Mayo Clinic, Rochester, $M N, U S A$

Phosphaturic mesenchymal tumors typically cause paraneoplastic osteomalacia, chiefly as a result of FGF23 secretion. In a prior study, we identified FN1-FGFR1 fusion in 9 of 15 phosphaturic mesenchymal tumors. In this study, a total of 66 phosphaturic mesenchymal tumors and 7 tumors resembling phosphaturic mesenchymal tumor but without known phosphaturia were studied. A novel FN1-FGF1 fusion gene was identified in two cases without FN1-FGFR1 fusion by RNA sequencing and cross-validated with direct sequencing and western blot. Fluorescence in situ hybridization analyses revealed FN1-FGFR1 fusion in 16 of 39 (41\%) phosphaturic mesenchymal tumors and identified an additional case with FN1-FGF1 fusion. The two fusion genes were mutually exclusive. Combined with previous data, the overall prevalence of FN1-FGFR1 and FN1-FGF1 fusions was $42 \%(21 / 50)$ and $6 \%(3 / 50)$, respectively. FGFR1 immunohistochemistry was positive in $82 \%(45 / 55)$ of phosphaturic mesenchymal tumors regardless of fusion status. By contrast, 121 cases of potential morphologic mimics (belonging to 13 tumor types) rarely expressed FGFR1, the main exceptions being solitary

E-mail: leejenchieh@ntuh.gov.tw or Folpe.Andrew@mayo.edu

${ }^{21}$ These authors contributed equally to this work.

Received 12 April 2016; revised 3 June 2016; accepted 3 June 2016; published online 22 July 2016 


\begin{abstract}
fibrous tumors (positive in $40 \%$ ), chondroblastomas (40\%), and giant cell tumors of bone (38\%), suggesting a possible role for FGFR1 immunohistochemistry in the diagnosis of phosphaturic mesenchymal tumor. With the exception of one case reported in our prior study, none of the remaining tumors resembling phosphaturic mesenchymal tumor had either fusion type or expressed significant FGFR1. Our findings provide insight into possible mechanisms underlying the pathogenesis of phosphaturic mesenchymal tumor and imply a central role of the FGF1-FGFR1 signaling pathway. The novel FN1-FGF1 protein is expected to be secreted and serves as a ligand that binds and activates FGFR1 to achieve an autocrine loop. Further study is required to determine the functions of these fusion proteins.
\end{abstract}

Modern Pathology (2016) 29, 1335-1346; doi:10.1038/modpathol.2016.137; published online 22 July 2016

Phosphaturic mesenchymal tumor is a distinctive soft tissue or bone neoplasm that typically, but not always, presents with hypophosphatemia and tumor-induced osteomalacia as a paraneoplastic syndrome. ${ }^{1,2}$ This syndrome appears to be mediated through tumor secretion of phosphatonins such as fibroblast growth factor 23 (FGF23, encoded by the FGF23 gene), secreted frizzled-related protein 4 (SFRP4), and matrix extracellular phosphoglycoprotein (MEPE), of which FGF23 is the best studied. ${ }^{3-7}$ The mechanisms underlying the tumorigenesis of phosphaturic mesenchymal tumor and its secretion of those phosphatonins remain obscure. Our recent identification of FN1-FGFR1 fusion gene in 9 of 15 phosphaturic mesenchymal tumors, with frequent overexpression of FGF1, has started to shed light on the genetic underpinnings of phosphaturic mesenchymal tumor and suggests a central role for fibroblast growth factors (FGFs)-fibroblast growth factor receptor 1 (FGFR1) autocrine/paracrine loop. ${ }^{8}$ However, we were previously unable to identify this FN1-FGFR1 fusion in roughly half of studied phosphaturic mesenchymal tumors, suggesting the existence of other tumorigenic mechanisms in these lesions. For this reason, we studied a larger group of well-characterized phosphaturic mesenchymal tumors for FN1-FGFR1 and alternative fusions.

\section{Materials and methods}

\section{Tumor Samples}

Pathology samples of phosphaturic mesenchymal tumors and tumors resembling phosphaturic mesenchymal tumor, diagnosed between January 1997 and May 2016, were collected from the archives of National Taiwan University Hospital (Taipei, Taiwan), Mayo Clinic (Rochester, MN, USA), and Vancouver General Hospital (Vancouver, $\mathrm{BC}$, Canada), as well as the consultation files of the authors (ALF and J-CL). A total of 73 tumors from 72 patients were collected, including 57 new patients in addition to the 15 previously studied cases. ${ }^{8}$ The clinical information is summarized in Supplementary Table S1. Three of the new cases (PMT-16, PMT-17 and PMT-50) had frozen tumor samples. The morphological diagnosis of all cases was confirmed by a soft tissue and bone pathologist (ALF or J-CL). All tumors showed classical features of phosphaturic mesenchymal tumor as previously described, including bland spindled cells embedded in a hyalinized to partially calcified matrix, with numerous small blood vessels, larger branching blood vessels and a variable component of osteoclasts and/or mature adipose tissue. All 65 patients classified as having a phosphaturic mesenchymal tumor either had (1) clinical symptoms and signs of osteomalacia, (2) elevated serum FGF23 levels, (3) high-level expression of FGF23 mRNA as demonstrated by RNAscope ${ }^{\circledR}$ chromogenic in situ hybridization (CISH), using previously published methods,${ }^{9,10}$ or (4) some combination of the first 3.

The seven tumors classified as 'tumors resembling phosphaturic mesenchymal tumor' showed identical morphologic features, but did not have documented osteomalacia, elevated serum FGF23, or positive FGF23 CISH. Among this group, one case (PMT-3) was previously found to contain the FN1-FGFR1 fusion in our prior study. ${ }^{8}$ Two of these lesions showed no FGF23 expression by CISH, while the remaining five cases (including PMT-3) failed the CISH analysis, likely owing to decalcification.

Potential morphological mimics were also retrieved from the archives of National Taiwan University Hospital during 1995 and 2015, including solitary fibrous tumors, myopericytic tumors, giant cell tumors of bone and soft tissue, tenosynovial giant cell tumors, aneurysmal fibrous histiocytomas, aneurysmal bone cysts, osteosarcomas, ossifying fibromas, non-ossifying fibromas, chondroblastomas, and osteoblastomas (10 cases each), as well as seven mesenchymal chondrosarcomas and four soft tissue chondromas with osteoclastic giant cells.

This research was approved by the research ethics boards in the respective institutions.

\section{RNA Sequencing and Data Analysis}

Total RNA was extracted from the three frozen tumor samples and subjected to RNA sequencing as previously described. ${ }^{8}$ Of note, the RNA of PMT-16 (2620 ng) and PMT-17 (1750 ng) did not pass the minimum requirement in amount $(5 \mu \mathrm{g})$ and was therefore pooled together with the RNA of a vascular 
neoplasm sample (630 ng) from another patient before being subjected to RNA sequencing. The raw data of the RNA sequencing results were uploaded to the NCBI SRA repository (accession numbers: SRX1560738 for PMT-50 and SRX1560739 for PMT-16 and PMT-17) and analyzed as previously described. ${ }^{8}$ In brief, detection of fusion genes was performed using TopHat software. ${ }^{11}$ UCSC human genome hg19 was used as the mapping reference. Gene expression was measured in fragments per kilobase of transcript per million mapped reads using Cufflinks (version 2.2.1) or RSEM software. A panel of 31 genes focusing on FGFs, FGFRs, and phosphatonins were used to generate a heat map as previously described. ${ }^{8}$ Multidimensional scaling plot and principal component analysis plot were generated with EdgeR and DESeq software, respectively, to determine the differential global expression between samples. ${ }^{12}$

\section{Validation of Fusion Genes and Proteins with PCR, Direct Sequencing, and Western Blot}

To validate the fusions identified with RNA sequencing, the remaining RNA from frozen samples and genomic DNA from archived samples were subjected to RT-PCR and PCR, respectively, as previously described, ${ }^{8}$ using specific primers (Supplementary Table S2), followed by Sanger sequencing. The fusion protein was characterized with western blot as previously described. ${ }^{8}$ Primary antibodies against fibronectin (1:1000 dilution; Abcam, Cambridge, UK), FGF1 (clone B-3; 1:500 dilution; Santa Cruz Biotechnology, Dallas, TX, USA), and FGFR1 (clone D8E4; 1:1000 dilution; Cell Signaling Technology, Danvers, MA, USA) were used.

\section{Fluorescence In Situ Hybridization}

FISH probes were synthesized by Cytotest (Rockville, MD, USA). The FN1-FGFR1 Tri-color Fusion/Translocation FISH Probe Kit had the $5^{\prime}$ region of the FN1 gene, the $5^{\prime}$ region of the FGFR1 gene, and $3^{\prime}$ region of the FGFR1 gene labeled with CytoAqua, CytoGreen, and CytoOrange fluorophores, respectively. The FGF1 Break-apart Probe Kit had the $5^{\prime}$ and $3^{\prime}$ regions of the FGF1 gene labeled with CytoOrange and CytoGreen, respectively. The reader is referred to the manufacturer's website for the details of the probe maps. Interphase FISH analyses were performed according to protocols and interpretation criteria as previously described. ${ }^{8}$ Selected cases were also analyzed using a DeltaVision deconvolution microscope (Applied Precision, South El Monte, CA, USA) as previously described. ${ }^{13}$

\section{Immunohistochemistry}

Immunohistochemistry was performed in a single pathology laboratory following standardized protocols. Briefly, tissue slides were subjected to heat- induced epitope retrieval ( $0.01 \mathrm{M}$ citrate buffer, $\mathrm{pH}$ 6.0) and immunohistochemistry was performed using primary antibodies against FGFR1 (clone D8E4; 1:500 dilution; Cell Signaling) and FGF1 (clone B-3; 1:20 dilution; Santa Cruz), followed by detection with the ultraView Universal DAB Detection Kit (Ventana Medical Systems, Tucson, AZ, USA). All phosphaturic mesenchymal tumor samples with multiple remaining slides were subjected to FGFR1 immunostaining. All cases with FGF1 translocation, 13 with FN1-FGFR1 fusion, 13 without fusion, and 13 with unknown fusion status were immunostained for FGF1. FGFR1 immunohistochemistry was also performed on 121 cases of potential morphologic mimics of phosphaturic mesenchymal tumor, as detailed above. Score 0 was assigned to cases showing overall negative staining (score 0); score $1+$ to focal (ie, in 1-50\% tumor cells) weak staining; score $2+$ to focal strong or diffuse (ie, $>50 \%$ tumor cells) weak staining; and score $3+$ to diffuse strong staining. Supplementary Figure S1 demonstrates various staining intensities. The scoring was performed by two soft tissue pathologists (J-CL and C-FL) independently; in case of disagreement, they were joined by a senior soft tissue pathologist $(\mathrm{H}-\mathrm{YH})$ to reach a consensus by a majority vote.

\section{Results}

\section{RNA Sequencing}

Novel FN1-FGF1 fusion genes were identified in both samples subjected to RNA sequencing. For PMT-50, the fusion transcripts joined the $3^{\prime}$ end of exon 24 of the FN1 gene with the intra-exonic region of FGF1 exon 2 (Figure 1a). Two major alternative splicing variants were detected, both of which were in-frame. Both breakpoints on the FGF1 part were located immediately $3^{\prime}$ to the splice acceptors (AG sequence), which were 30 nucleotides apart. Of note, infrequent reads were found spanning another fusion junction that joined the $F N 1$ intron 24 region to a nucleotide position in exon 2 of FGF1 which was 7 and 37 base pairs, respectively, $5^{\prime}$ to the major FGF1 breakpoints. Given the involvement of intronic sequence and expected frameshift, this minor fusion product likely represented the unspliced mRNA precursor, thus reflecting the genomic DNA breakpoint as we have previously suggested. ${ }^{8}$ All of the mRNA and genomic DNA fusion junctions were confirmed with Sanger sequencing following RT-PCR and DNA PCR (Figure 1b).

For the pooled PMT-16 and PMT-17 sample, two major alternative $F N 1-F G F 1$ in-frame fusion variants were also detected. The breakpoints found in FGF1 were identical to those observed in PMT-50. The breakpoint for the FN1 gene was located at the $3^{\prime}$ end of exon 26. By using Integrative Genomics Viewer (https://www.broadinstitute.org/igv/), minor reads 
were disclosed spanning the presumed genomic fusion junction, which fused the FN1 intron 26 region with exon 2 of $F G F 1$ (the breakpoint was 6-bp $5^{\prime}$ to that observed in PMT-50). In addition, a novel FN1-FGFR1 fusion variant (exon 26 to exon 4) and its corresponding genomic fusion junction (intron 26

a

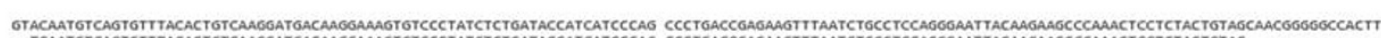
TCAATOTCAGTOTTTACACTGTCAAGGATGACAAGGAAAOTOTCCCTATCTCTOATACCATCATCCCAG CCCTOACC CAGAAGTTTAATCTOCCTCCAGGGAATTACAAGAAGCCCCAACTCCTCTACT GTAG

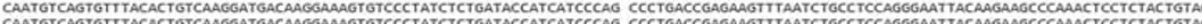

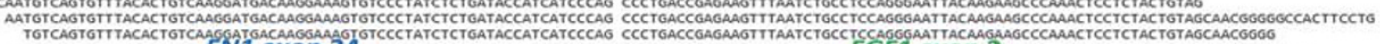

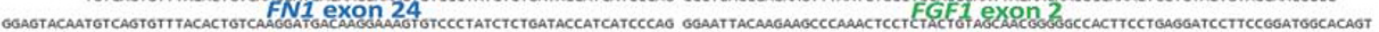
GTACAATGTCAGTOTTTACACTGTCAAGGATGACAAGSAAAGTOTCCCTATCTCT GATACCATCATCCCAG GGAATTACAAGAAGCCCAAACTCCTCTACTOTAGCAACGOGGOCCACTTCCT GAGGATCCTTCCOGATGOCACAGTGGA

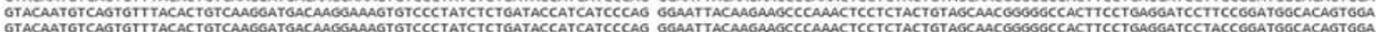
GACATTOCOOTOTTACACTOTCAGCAT

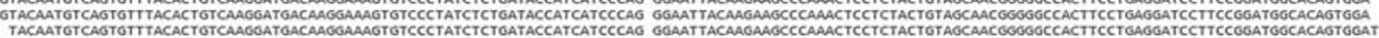

orre (925) प्र

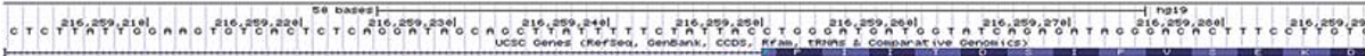

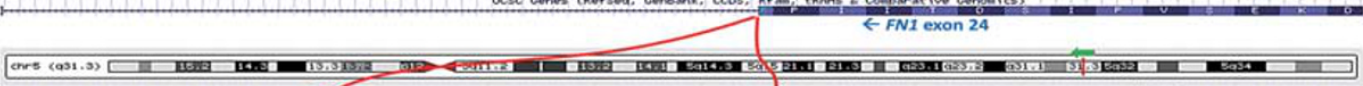

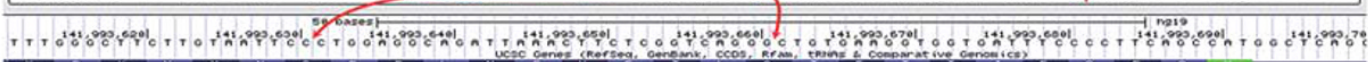
$\leftarrow$ FGF1 exon 2

b

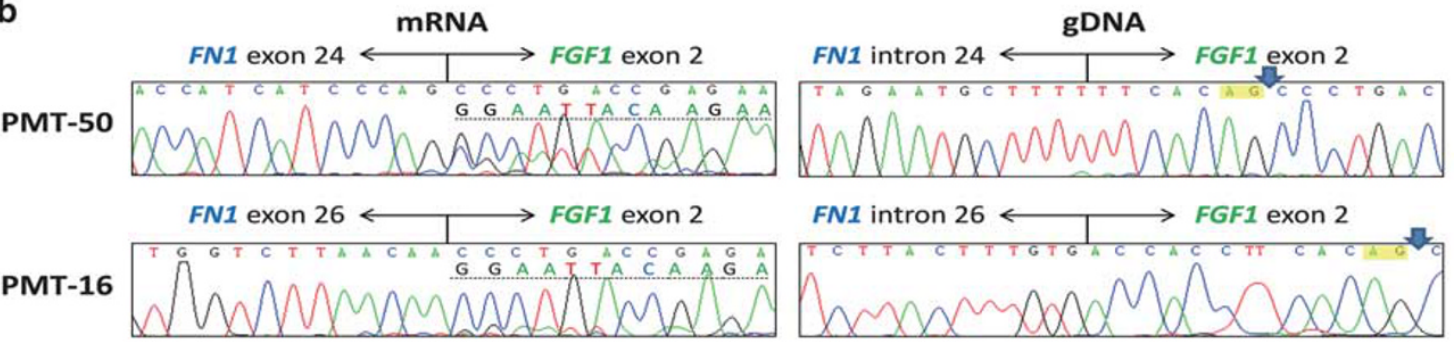
FN1 exon $26 \longleftrightarrow$ FGFR1 exon 4
PMT-17

C

FN1-FGF1 chimeric protein $\mathrm{NH} 2$

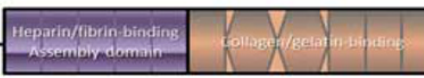

홍 $\infty \frac{0}{5}$

ป

FGF1
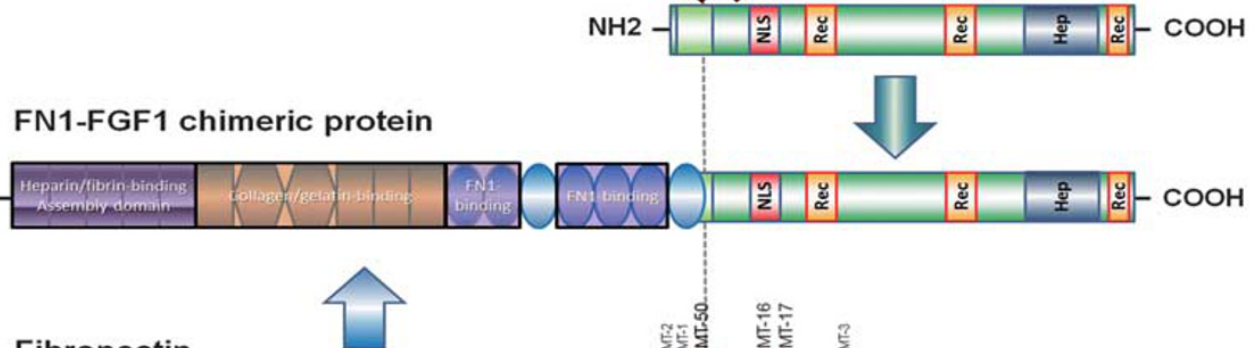

Fibronectin
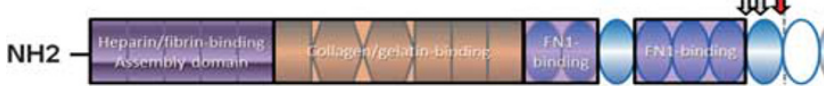

密言

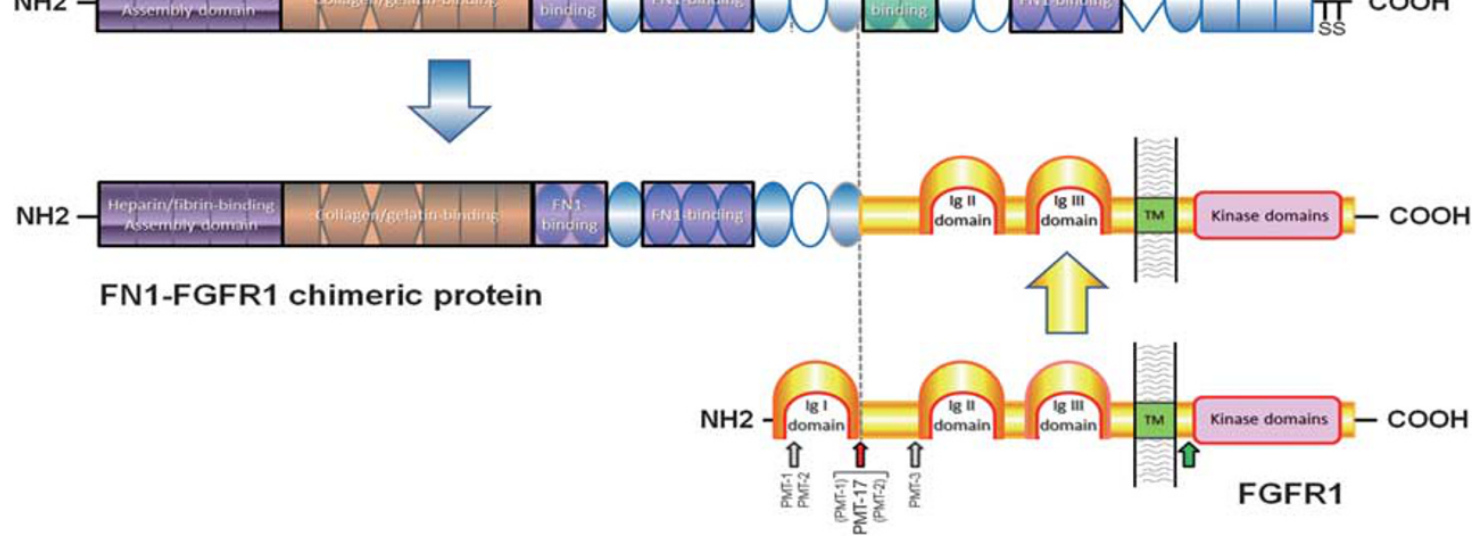


to intron 3) were also detected. By Sanger sequencing, the FN1-FGF1 and FN1-FGFR1 fusion genes were confirmed to originate from PMT-16 and PMT-17, respectively (Figure 1b).

The fusion transcripts and respective read counts disclosed by RNA sequencing are summarized in Table 1. The predicted domains retained in the newly found FN1-FGF1 and FN1-FGFR1 proteins are illustrated in Figure 1c. The predicted molecular weights of these fusion proteins are 155 and $156 \mathrm{kDa}$ in PMT-50, 171 and $172 \mathrm{kDa}$ in PMT-16, and $235 \mathrm{kDa}$ in PMT-17.

Regarding the expression signature of the 31-gene panel, ${ }^{8}$ PMT-50 clustered with PMT-1 and PMT-2 (both with FN1-FGFR1 fusion) in the hierarchical clustering analysis (Supplementary Figure S2). Specifically, like the other phosphaturic mesenchymal tumors, PMT-50 expressed relatively high levels of FN1 (918 fragments per kilobase of transcript per million mapped reads and ranking $=187 / 23619$ ), FGFR1 (158 and 455/23619), FGF1 (159 and 453/23619), FGF23 (444 and 265/23619), MEPE (1068 and 172/23619), and SFRP4 (926 and 186/23619), whereas the other members in the FGFR and FGF families as well as the $K L$ gene (coding for $\alpha$-Klotho) were expressed at low levels. Furthermore, PMT-50 also showed a similar global expression profile to those of PMT-1 and PMT-2 in EdgeR multidimensional scaling plot and DESeq principal component analysis plot (Supplementary Figure S2), indicating a high level of homogeneity of fusion-positive phosphaturic mesenchymal tumors, regardless of fusion type.

\section{Western Blot}

PMT-50 had remaining frozen tissue and was subjected to western blot analysis (Figure 2). A band of protein at estimated $180-200 \mathrm{kDa}$ was stained with both fibronectin (encoded by FN1) and FGF1 immunoblotting, removed from their respective normal molecular weights ( $>250$ and $17 \mathrm{kDa}$ ), indicating the presence of the FN1-FGF1 chimeric protein. Normal fibronectin was expressed at a high level. There was no detectable expression of normal FGF1; by contrast, PMT-2, which harbored the FN1-FGFR1 fusion ${ }^{8}$ and served as a control case here, seemed to express normal FGF1. A low level of FGFR1 protein of normal molecular weight was also detected in PMT-50.

\section{Fluorescence In Situ Hybridization}

A total of 61 tumors from 60 patients were analyzed with FN1-FGFR1 tricolor FISH, including 4 tumors with known FN1-FGFR1 fusion (PMT-1, PMT-2, PMT-5, and PMT-17) as positive controls ${ }^{8}$ as well as 6 tumors resembling phosphaturic mesenchymal tumor. Interpretable results were obtained from 44 tumors. In all, 16 of the 39 (41\%) phosphaturic mesenchymal tumors were positive for FN1-FGFR1 fusion, including the 4 positive control cases (Figure 3a). Interestingly, FN1-FGFR1 fusion without split FGFR1 signals (ie, with juxtaposition of signals of all three colors) was noted in 5 of the 16 tumors (Figure $3 \mathrm{~b}$ and $\mathrm{c}$ ), suggestive of insertion of FN1 gene into the FGFR1 gene or other complex forms of rearrangement. None of the other tumors, including all of the five successfully analyzed tumors resembling phosphaturic mesenchymal tumor, had the FN1-FGFR1 fusion or FGFR1 rearrangement. Of the 17 tumors in which FISH analyses failed, 15 were bone tumors (likely decalcified).

FGF1 FISH was performed on 65 tumors, including all 34 tumors negative for the FN1-FGFR1 fusion and 14 randomly selected FN1-FGFR1-positive cases. Seventeen tumors failed the analysis, all of which had also failed the FN1-FGFR1 FISH study. Of the remaining 48 tumors, 3 (PMT-16, PMT-50, and PMT-31) showed split FGF1 signals (Figure 3d). None of the 14 tumors with FN1-FGFR1 fusion or the 5 tumors resembling phosphaturic mesenchymal tumor had FGF1 rearrangement. To further determine whether the fusion partner of FGF1 gene in PMT-31 was also FN1, FISH was performed using a 1:1 mixture of FN1-FGFR1 and FGF1 probes. Nuclei harboring blue signals (for $5^{\prime} F N 1$ ) fused with green signals (for either $3^{\prime}$ FGF1 or 5' FGFR1) were observed (Figure 3e). Collectively, these results were highly suggestive of FN1-FGF1 fusion in PMT-31. See Supplementary Table S1 for details of FISH results.

\section{FGFR1 and FGF1 Immunohistochemistry}

A positive FGFR1 result, defined as score $2+$ or 3+, was observed in $82 \%(45 / 55)$ of phosphaturic mesenchymal tumors. Specifically, 94\% (17/18), $100 \%(3 / 3)$, $90 \%(18 / 20)$, and $50 \%(7 / 14)$ of phosphaturic mesenchymal tumors with the FN1-FGFR1 fusion, the FN1-FGF1 fusion, no fusion, and

Figure 1 (a) The representative alignment of reads covering the two major fusion points in PMT-50 and the respective chromosomal and exonic locations (data source: http://genome.ucsc.edu/). ${ }^{46}$ (b) Validation of the RNA sequencing results by Sanger sequencing following RT-PCR of mRNA or PCR of genomic DNA (gDNA). Immediately $3^{\prime}$ to the fusion junctions, the FGF1 sequences underscored by dotted lines indicate the alternatively spliced transcript, shown in the electropherogram as minor peaks. The blue arrows in the genomic DNA sequences indicate the closer mRNA breakpoints of FGF1, immediately 3' to the splice acceptor AG sequence. (c) The schematic diagram of the expected domains retained in the chimeric proteins (FN1-FGF1 identified in PMT-50 and FN1-FGFR1 in PMT-17). Smaller arrows indicate the breakpoints in respective cases. By contrast, the most common breakpoint of FGFR1 observed in other tumor types is located in the cytosolic juxtamembranous domain (green arrow). Hep, heparin-binding domain; NLS, nuclear localization signal; Rec, receptorbinding domain; TM, transmembrane domain. Refer to Singh et al. ${ }^{28}$ and Atlas of Genetics and Cytogenetics in Oncology and Haematology website (http://atlasgeneticsoncology.org/Genes/FGFR1113.html) for details of the protein domains. 
Table 1 Summary of the RNA sequencing results

\begin{tabular}{|c|c|c|c|c|c|}
\hline Case & Fusion junctions & Exon & $\begin{array}{l}\text { Spanning } \\
\text { reads }^{\mathrm{a}}\end{array}$ & $\begin{array}{l}\text { Spanning } \\
\text { mate pairs }\end{array}$ & $\begin{array}{l}\text { Mate pairs where one } \\
\text { end spans a fusion }{ }^{\mathrm{C}}\end{array}$ \\
\hline \multirow[t]{3}{*}{ PMT-50 } & chr2:216259251 > chr5:141993661 & $F N 1$ exon $24>F G F 1$ exon 2 & 374 & 10 & 75 \\
\hline & chr2:216259251 > chr5:141993631 & $F N 1$ exon $24>F G F 1$ exon 2 & 206 & 10 & 46 \\
\hline & $\operatorname{chr} 2: 216258567>\operatorname{chr} 5: 141993668$ & $F N 1$ intron $24>F G F 1$ exon $2^{\mathrm{d}}$ & 16 & 10 & 6 \\
\hline \multirow[t]{3}{*}{ PMT-16 } & $\operatorname{chr} 2: 216256355>\operatorname{chr} 5: 141993661$ & $F N 1$ exon $26>F G F 1$ exon 2 & 460 & 5 & 46 \\
\hline & $\operatorname{chr} 2: 216256355>\operatorname{chr} 5: 141993631$ & $F N 1$ exon $26>F G F 1$ exon 2 & 39 & 5 & 10 \\
\hline & chr2:216255473 > chr5:141993674 & $F N 1$ intron $26>F G F 1$ exon $2^{\mathrm{d}}$ & & e & \\
\hline \multirow[t]{2}{*}{ PMT-17 } & chr2:216256355 > chr8:38285953 & FN1 exon $26>F G F R 1$ exon 4 & 326 & 3 & 42 \\
\hline & chr2:216255371 > chr8:38286974 & $F N 1$ intron $26>F G F R 1$ intron $3^{\mathrm{d}}$ & 13 & 3 & 3 \\
\hline
\end{tabular}

${ }^{\mathrm{a}}$ The numbers of reads that span a fusion point. ${ }^{\mathrm{b}}$ The number of pairs of reads where one read maps entirely on the left and the other read maps entirely on the right of the fusion breakpoint. ${ }^{\mathrm{C}}$ The number of spanning mate pairs where one end spans a fusion (reads spanning fusion with only a few bases are included). ${ }^{\mathrm{d}}$ Presumed genomic DNA fusion junction. ${ }^{\mathrm{e}}$ Detected by using Integrative Genomics Viewer.
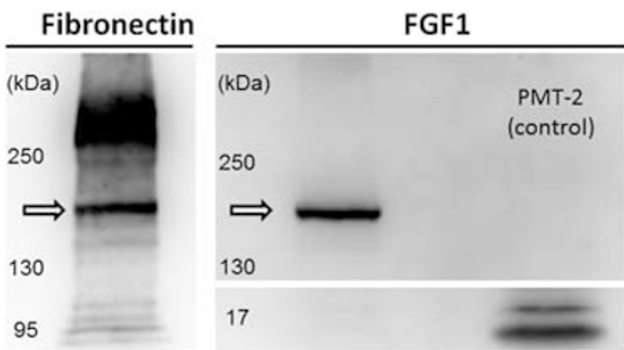

\begin{tabular}{l} 
FGFR1 \\
\hline (KDa) \\
250 \\
\\
130 \\
95 \\
72
\end{tabular}

Figure 2 Western blot analysis of PMT-50. The fusion protein (indicated with arrows), with a molecular size of estimated 180$200 \mathrm{kDa}$, is stained with both fibronectin and FGF1 immunoblotting. Normal fibronectin $(>250 \mathrm{kDa})$ is also expressed by the tumor. No conspicuous expression of normal FGF1 is observed in PMT-50, in contrast to PMT-2 (harboring FN1-FGFR1 fusion gene). Weak expression of normal FGFR1 (about $100 \mathrm{kDa}$ ) is also observed in PMT-50.

unknown fusion status, respectively, were positive. The staining pattern was chiefly cytoplasmic, whereas immunoreactivity in the areas of heavy matrix deposition was observed in seven cases regardless of the fusion status (Figure $4 \mathrm{a}-\mathrm{c}$ ). Of note, with the exception of PMT-3, none of the other five tumors resembling phosphaturic mesenchymal tumor was positive. In contrast, of the potential mimics, positive staining (mostly $2+$ ) was observed only in a minority of solitary fibrous tumors (40\%), chondroblastomas $(40 \%)$, giant cell tumors of bone $(38 \%)$, non-ossifying fibromas $(20 \%)$, osteosarcomas $(10 \%)$, and osteoblastomas $(10 \%)$.

The three phosphaturic mesenchymal tumors with the FN1-FGF1 fusion showed variable FGF1 immunoreactivity (scores $1+$ to $3+$ ). Of the other phosphaturic mesenchymal tumors evaluated, $38 \%(5 / 13)$, $77 \% \quad(10 / 13)$, and $69 \%$ (9/13) of phosphaturic mesenchymal tumors with the FN1-FGFR1 fusion, no fusion, and unknown status, respectively, showed at least $1+$ staining. The staining pattern was mainly cytoplasmic, whereas nuclear and extracellular staining was each observed in 15 cases, regardless of the fusion status (Figure $4 \mathrm{~d}-\mathrm{f}$ ). Table 2 and
Supplementary Table S1 summarize the immunohistochemistry results.

\section{Discussion}

Phosphaturic mesenchymal tumors are a distinctive type of mesenchymal tumor that typically causes osteomalacia through secreting circulating phosphatonins, particularly FGF23, leading to renal wasting of phosphate. ${ }^{3,4}$ Historically considered to be caused by a wide variety of tumor types, tumor-induced osteomalacia is now understood to be largely caused by a single histopathological entity (phosphaturic mesenchymal tumor) showing a spectrum of morphological appearances. ${ }^{1,2,14,15}$ This 'unifying concept' is supported by our recent discovery of the FN1-FGFR1 fusion gene in a significant subset of phosphaturic mesenchymal tumors, as well as by the results of the present study. ${ }^{8}$

In this study, we systematically characterized the prevalence of the FN1-FGFR1 fusion in a larger group of phosphaturic mesenchymal tumors. Combined with previous data, ${ }^{8} 42 \%$ (21/50) of phosphaturic mesenchymal tumors contained the FN1-FGFR1 fusion gene. Notably, 5 of 16 cases in this series showed co-localization of the $5^{\prime}$ region of FN1 with the $5^{\prime}$ and $3^{\prime}$ regions of FGFR1, suggestive of complex forms of rearrangement that would probably not be detected with FGFR1 break-apart FISH probes alone, as we have previously hypothesized. ${ }^{8}$ As exemplified by PMT-17, all the FN1-FGFR1 fusion-containing variants with known fusion junctions are expected to retain the extracellular FGF-binding domains and the transmembrane domain, different from most other FGFR1 fusion proteins. ${ }^{16}$ This feature implies a possible role for FGFs (particularly the highly expressed FGF23 and FGF1) in facilitating the activation of the FN1-FGFR1 protein, thereby creating an autocrine loop mechanism. In addition, the FN1-FGFR1 protein might require the transmembrane domain to be anchored on the membrane to avoid being secreted. This concept is potentially 
supported by a recently identified FN1-ALK oncoprotein, which contains the ALK transmembrane domain absent from other ALK fusion proteins. ${ }^{17,18}$
Additionally, a novel FN1-FGF1 fusion gene was identified in three phosphaturic mesenchymal tumors, all originating from bone. If we assume that
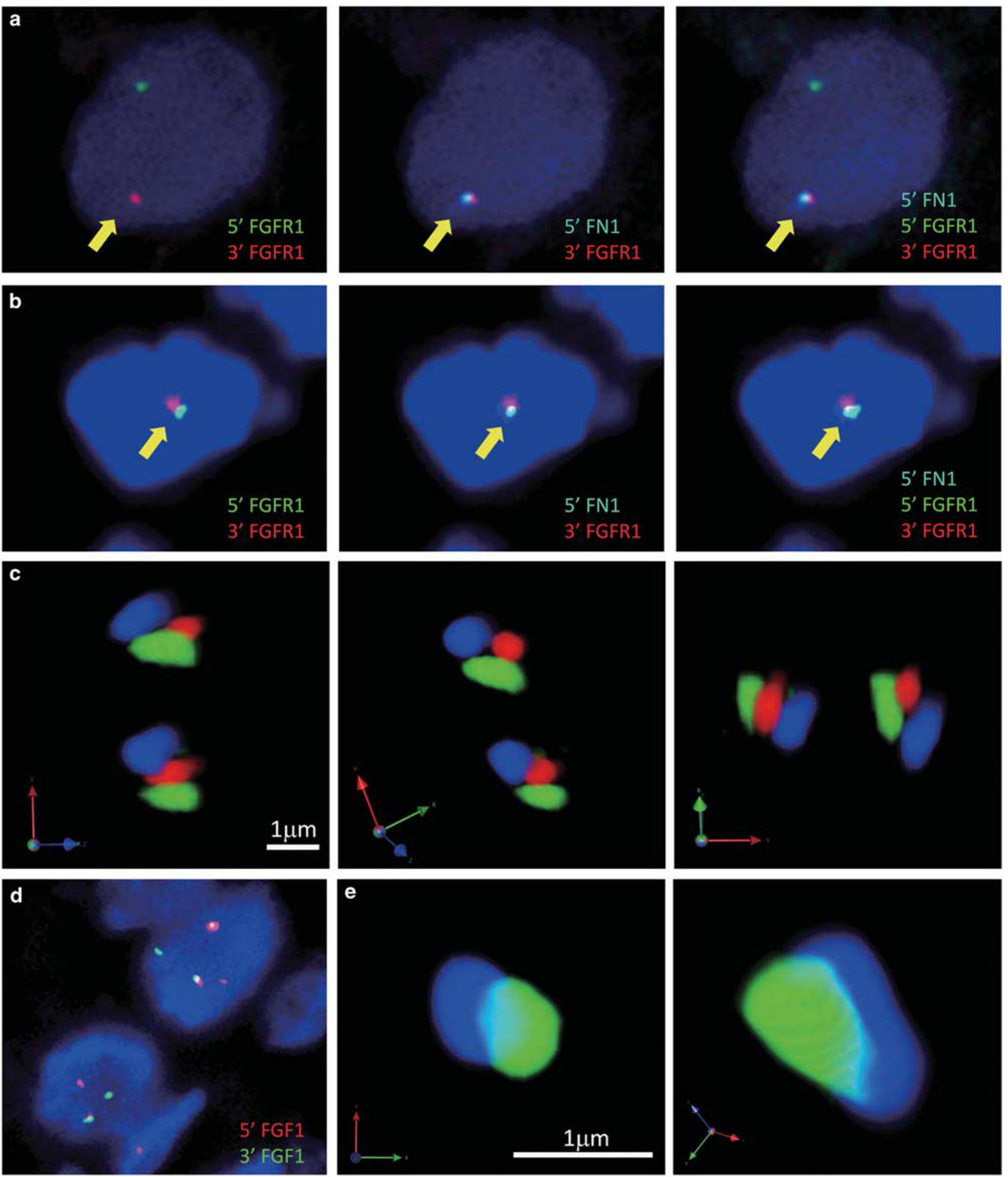

Figure 3 Examples of fluorescence in situ hybridization results. (a) PMT-19 shows juxtaposition of 5' FN1 and 3' FGFR1 signals, while the $5^{\prime}$ and $3^{\prime}$ ends of FGFR1 were broken apart. By contrast, PMT-42 (b) and PMT-5 (c, 3-dimensional reconstruction by deconvolution microscopy) reveal juxtaposition of signals of $5^{\prime} F N 1,5^{\prime} F G F R 1$, and 3' FGFR1, which implies more complex forms of rearrangement and could evade detection by FGFR1 break-apart probes alone. (d) PMT-50 harbors break-apart FGF1 gene. (e) By using mixed FN1-FGFR1 and FGF1 probes, FN1-FGF1 fusion (blue-green fusion) is disclosed in PMT-31. 

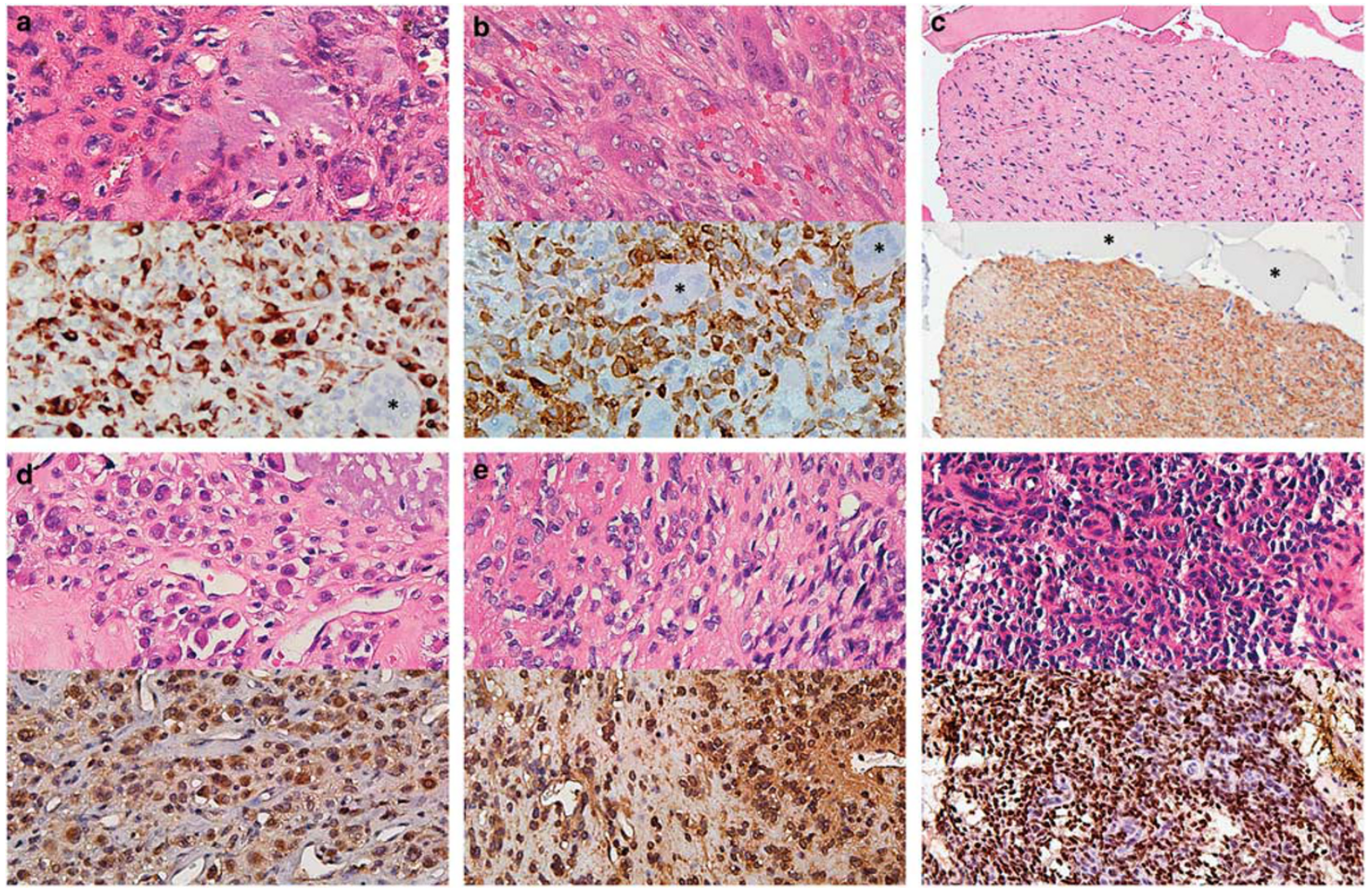

Figure 4 (a-c) FGFR1 immunohistochemistry (with corresponding hematoxylin and eosin staining) shows strong immunoreactivity in cases with (a: PMT-20) and without (b: PMT-23 and c: PMT-15) FN1-FGFR1 fusion, presenting mainly cytoplasmic staining pattern. Areas of heavy matrix deposition (c) also often present FGFR1 immunoreactivity. As internal controls, osteoclastic giant cells in (a and b), as well as skeletal muscle cells in (c), are negative (indicated with asterisks). (d-f) FGF1 immunohistochemistry (with corresponding hematoxylin and eosin staining) exhibits chiefly cytoplasmic staining whereas extracellular immunoreactivity (d: PMT-50 and e: PMT-58) or nuclear staining (f: PMT-47) is sometimes observed.

FN1-FGF1 and FN1-FGFR1 fusions are mutually exclusive, as suggested by the data from representative cases, then the estimated overall prevalence of FGF1 rearrangement and rearrangement of either gene in phosphaturic mesenchymal tumors is $6 \%$ $(3 / 50)$ and $48 \%(24 / 50)$, respectively. It is noteworthy that nearly half of decalcified tumors with unknown fusion status showed 2+ to $3+$ FGF1 immunoreactivity, implying that this fusion might be enriched in bone tumors, with its prevalence possibly underestimated by tissue decalcification. To our knowledge, the presence of gene fusions involving FGF1 has not been previously reported in human neoplasms, although overexpression of FGF1 has been implicated in a variety of carcinomas. ${ }^{19-22}$

Because both fibronectin (encoded by the FN1 gene) and FGF1 are secretory proteins, the FN1-FGF1 fusion protein is expected to be secreted, in keeping with the immunohistochemistry findings. The fusion protein contains nearly the entirety of FGF1 and, when secreted at high levels, could presumably function like normal FGF1 in excess. FGF1 protein is a crucial ligand for all FGFRs. ${ }^{23}$ It acts as a potent mitogen of fibroblasts and is involved in critical bio- logical functions including development, morphogenesis, and angiogenesis. ${ }^{24,25}$ The expected retention of the nuclear localization sequence of FGF1 in this fusion protein is noteworthy, as the receptormediated internalization and nuclear localization sequence-dependent nuclear translocation of FGF1 is crucial for FGF1-induced mitogenic effects. ${ }^{26,27}$ Of interest, focal nuclear staining of FGF1 was observed in some phosphaturic mesenchymal tumors regardless of the fusion status, suggesting that the biological functions of nuclear FGF1 might be in effect in these tumors.

As in the FN1-FGFR1 fusion gene, the FN1 gene probably provides its transcriptionally active promoter to drive the expression of FN1-FGF1 protein. However, the invariable and extensive involvement of FN1 as the fusion partner gene in phosphaturic mesenchymal tumor implies a more important role for the FN1 part. Fibronectin, an essential extracellular matrix molecule, likely retains in the fusion protein the domains that can bind heparin, fibrin, and collagen, as well as fibronectin itself. Some of these domains are responsible for fibronectin selfassembly and fibril formation. ${ }^{28,29}$ Both FN1-FGFR1 and FN1-ALK fusion proteins could utilize the 
Table 2 The summary of FGFR1 and FGF1 immunohistochemistry results

\begin{tabular}{|c|c|c|c|c|c|c|c|c|c|c|}
\hline \multirow{2}{*}{ Diagnosis } & \multirow{2}{*}{ Subgroup } & \multicolumn{5}{|c|}{$F G F R 1^{\mathrm{a}}$} & \multicolumn{4}{|c|}{$F G F 1^{\text {a }}$} \\
\hline & & 0 & $1+$ & $2+$ & $3+$ & Positive $(2+/ 3+)$ & 0 & $1+$ & $2+$ & $3+$ \\
\hline \multirow[t]{5}{*}{ PMT } & FN1-FGFR1 & - & $1(6)$ & $9(50)$ & $8(44)$ & $17(94)$ & $8(62)$ & $1(8)$ & $4(31)$ & - \\
\hline & $F N 1-F G F 1$ & - & - & $2(67)$ & $1(33)$ & $3(100)$ & - & $1(33)$ & $1(33)$ & $1(33)$ \\
\hline & Fusion negative & $1(5)$ & $1(5)$ & $9(45)$ & $9(45)$ & $18(90)$ & $3(23)$ & $4(31)$ & $5(38)$ & $1(8)$ \\
\hline & Fusion unknown & $5(36)$ & $2(14)$ & $4(29)$ & $3(21)$ & $7(50)$ & $4(31)$ & $3(23)$ & $3(23)$ & $3(23)$ \\
\hline & Overall & $6(11)$ & $4(7)$ & $24(44)$ & $21(38)$ & $45(82)$ & $15(36)$ & $9(21)$ & $13(31)$ & $5(12)$ \\
\hline \multirow{6}{*}{$\begin{array}{l}\text { TRPMT } \\
\text { SFT } \\
\text { Chondroblastoma } \\
\text { GCT }\end{array}$} & & 3 & 2 & $1^{\mathrm{b}}$ & - & $1^{\mathrm{b}}$ & $1^{\mathrm{b}}$ & - & - & - \\
\hline & & 3 & 3 & 3 & 1 & 4 & & & & \\
\hline & & 2 & 4 & 4 & - & 4 & & & & \\
\hline & Bone & 1 & 4 & 2 & 1 & 3 & & & & \\
\hline & Soft tissue & 2 & - & - & - & - & & & & \\
\hline & Overall & 3 & 4 & 2 & 1 & 3 & & & & \\
\hline \multirow{4}{*}{$\begin{array}{l}\text { NOF } \\
\text { OGS }\end{array}$} & & 3 & 5 & 2 & - & 2 & & & & \\
\hline & Conventional type & 6 & 2 & - & - & - & & & & \\
\hline & Low-grade type & 1 & - & 1 & - & 1 & & & & \\
\hline & Overall & 7 & 2 & 1 & - & 1 & & & & \\
\hline \multirow{4}{*}{$\begin{array}{l}\text { Osteoblastoma } \\
\text { TSGCT }\end{array}$} & & - & 1 & 1 & - & 1 & & & & \\
\hline & Localized type & 5 & - & - & - & - & & & & \\
\hline & Diffuse type & 4 & 1 & - & - & - & & & & \\
\hline & Overall & 9 & 1 & - & - & - & & & & \\
\hline \multirow{3}{*}{\multicolumn{2}{|c|}{$\begin{array}{l}\text { Aneurysmal FH } \\
\text { Chondroma }^{\mathrm{C}} \\
\text { ABC }\end{array}$}} & 5 & 5 & - & - & - & & & & \\
\hline & & 2 & 2 & - & - & - & & & & \\
\hline & & 8 & 2 & - & - & - & & & & \\
\hline \multirow[t]{4}{*}{ Pericytic tumor } & Myofibroma & 4 & - & - & - & - & & & & \\
\hline & Myopericytoma & 3 & - & - & - & - & & & & \\
\hline & Sinonasal HPC & 3 & - & - & - & - & & & & \\
\hline & Overall & 10 & - & - & - & - & & & & \\
\hline \multirow{2}{*}{\multicolumn{2}{|c|}{$\begin{array}{l}\text { OF } \\
\text { M-CHS }\end{array}$}} & 10 & - & - & - & - & & & & \\
\hline & & 7 & - & - & - & - & & & & \\
\hline
\end{tabular}

Abbreviations: ABC, aneurysmal bone cyst; FH, fibrous histiocytoma; GCT, giant cell tumor of bone or soft tissue; HPC, hemangiopericytoma; M-CHS, mesenchymal chondrosarcoma; NOF, non-ossifying fibroma; OF, ossifying fibroma; OGS, osteosarcoma; PMT, phosphaturic mesenchymal tumor; SFT, Solitary fibrous tumor; TRPMT, tumor resembling PMT; TSGCT, tenosynovial giant cell tumor.

${ }^{\mathrm{a}}$ Numbers in parentheses represent the percentages.

bPMT-3.

${ }^{\mathrm{C}}$ Soft tissue chondroma with osteoclastic giant cells

fibronectin self-assembly mechanism to achieve the ligand-independent activation of respective tyrosine kinases. Moreover, it has been shown that with the assistance of heparin, either dimerization of FGF1 or of FGFRs precedes the FGF1-FGFR binding in a 2:2 ternary fashion. ${ }^{30-32}$ Therefore, both the self-assembly and heparin-binding qualities of the fibronectin part might facilitate the FGF1-FGFR interaction.

It is noteworthy that a novel FN1-EGF fusion gene has very recently been identified in calcifying aponeurotic fibroma, another rare soft tissue tumor characterized by production of unusual calcified matrix. ${ }^{33}$ Both the FN1-FGF1 and FN1-EGF fusions involve secretory growth factors and may possibly drive tumorigenesis via autocrine mechanisms in mesenchymal tumors that typically show calcified matrix deposition, suggesting a common theme of fibronectin expression and interaction with extracellular matrix and growth factors in tumor formation. Interestingly, a reversely chimeric FGF1-FN1 protein engineered to fuse the cell-binding domain of fibronectin to the C-terminus of full-length FGF1 protein has been demonstrated to exert superior adhesion, mitogenesis, and differentiation effects on osteoblast-like cells, as compared with fibronectin or FGF1 alone. ${ }^{34}$ However, as the Arg-Gly-Asp sequence responsible for the cell-binding ability of fibronectin is not expected to be retained in the FN1-FGF1 fusion protein in PMT-16 or PMT-50, the relevance of these findings to the oncogenic properties of FN1-FGF1 in phosphaturic mesenchymal tumors remains to be determined.

Roughly half of phosphaturic mesenchymal tumors in this study did not contain either fusion gene. Although it is likely that other driver mutations underlie the pathogenesis of these tumors, their frequent expression of FGFR1 still suggests a possible role for FGFR1 signaling. The notable difference between the results of FISH analyses on archived tissue and RNA sequencing using fresh frozen tissue (where all five tumors had either fusion) may reflect the inherently lower sensitivity of FISH on archival tissue; alternatively, it might suggests the existence of cryptic fusions or fusions in a subpopulation of tumor cells that could evade detection by FISH. 


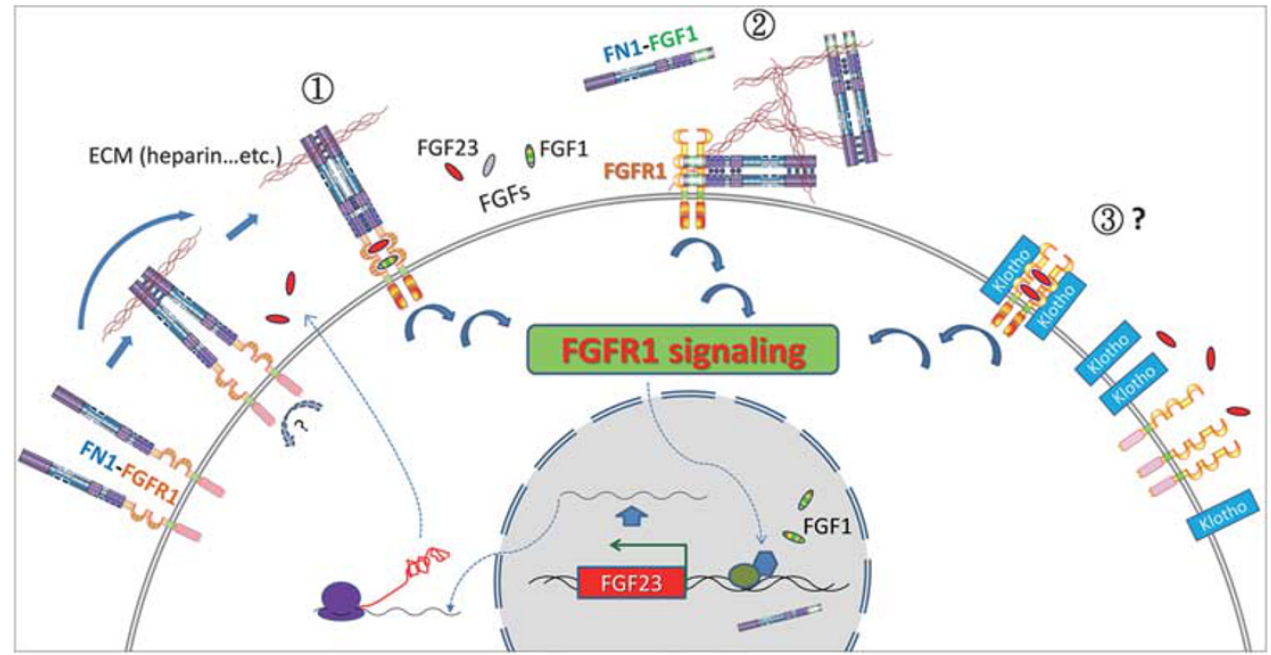

Figure 5 The schematic diagram of proposed tumorigenesis mechanisms of phosphaturic mesenchymal tumor. (1) FN1-FGFR1 proteins might use the self-assembly and fibronectin-binding domains of the FN1 part to dimerize, perhaps with the assistance of extracellular matrix (such as heparin). The FGFs (eg, FGF1 and FGF23) secreted by the tumor cells might bind the ligand-binding domains of the FGFR1 part to facilitate the dimerization and activation of the fusion protein. (2) FN1-FGF1 protein is probably secreted and, with the assistance of heparin, might dimerize and bind the membranous FGFR1 in a 2:2 ternary fashion. (3) $\alpha$-Klotho serves as an obligatory co-receptor for the FGF23-FGFR1 binding and, when overexpressed in osteocytes or their FGF23-secreting precursors, might allow FGF23 to activate FGFR1. The three pathways converge in the activation of FGFR1 signaling, which might upregulate the expression of FGF23. The nuclear translocation of FGF1 proteins, in either native or chimeric form, might also have a role in the tumorigenesis.

Among these fusion-negative cases, PMT-61 is particularly insightful. This patient has a de novo germline translocation involving the region upstream of the $K L$ (Klotho) gene, which probably accounted for her congenital elevation of plasma $\alpha$-Klotho level and hypophosphatemic rickets. ${ }^{35}$ At age 30 , two tumors were found in her bilateral mastoid bones, confirmed histopathologically to be phosphaturic mesenchymal tumors, and shown by CISH to express elevated levels of FGF23. Both tumors were fusion-negative and the one tested by immunohistochemistry showed diffuse and weak expression of FGFR1 and FGF1. $\alpha$-Klotho is an obligatory co-receptor for FGF23-FGFR1 binding, and its expression is mostly limited to renal tubules, choroid plexus, and parathyroid gland under normal physiologic condition. ${ }^{36}$ It is possible that the systemic overexpression of $\alpha$-Klotho in this patient might set the stage for an FGF23-FGFR1 autocrine loop to be established in FGF23-secreting cells, such as osteocytes. In addition, the FGFR1 signaling could mediate the upregulation of FGF23 in osteocytes, ${ }^{37-41}$ thereby exerting a positive feedback mechanism, with creation of a self-sufficient cellular environment and eventual formation of highly FGF23-secreting tumors, ie, phosphaturic mesenchymal tumors. This could explain why this patient would have two such rare tumors. Intriguingly, a phosphaturic mesenchymal tumor with $\alpha$-Klotho expression was recently reported, in contrast to our findings. ${ }^{42}$ It remains to be determined whether some phosphaturic mesenchymal tumors could exploit aberrant $\alpha$-Klotho expression as a tumorigenic driver.

Figure 5 illustrates in schematic form our hypothesized mechanisms of phosphaturic mesenchymal tumor pathogenesis based on the current genetic findings, in which FGFR1 signaling serves as a convergent pathway accounting for both tumor initiation and FGF23 overproduction.

With the notable exception of PMT-3, those mesenchymal tumors that morphologically closely resembled phosphaturic mesenchymal tumors, but lacked evidence of osteomalacia or FGF23 production, were fusion-negative and showed at best minimal (1+) FGFR1 expression. It should be emphasized that clinical information on these patients was quite limited, and we do not know whether these patients truly lacked osteomalacia, whether we were not provided with this information, or even whether these patients had been evaluated for signs and symptoms of tumor-induced osteomalacia. Expression of FGF23 by RT-PCR has been previously shown by Bahrami et al. ${ }^{43}$ in similar tumors, suggesting that at least a subset of these lesions do in fact represent phosphaturic mesenchymal tumors. Further study will be necessary to determine whether other cases of 'tumor resembling phosphaturic mesenchymal tumor' are truly related to phosphaturic mesenchymal tumor, or simply morphological mimics.

Among the other mesenchymal tumors studied, solitary fibrous tumor, giant cell tumor of bone, and chondroblastoma had relatively high frequencies of FGFR1 expression. Fortunately, the high prevalence of NAB2-STAT6 fusion (with nuclear STAT6 expression) in solitary fibrous tumor and $H 3 F 3 A / B$ hotspot mutations in giant cell tumor and chondroblastoma help to distinguish them from phosphaturic mesenchymal tumors. ${ }^{44,45}$ With those three notable exceptions, high-level expression of FGFR1 seems to be 
relative sensitive and specific for phosphaturic mesenchymal tumor, and may be of some value as an adjunctive test in selected cases.

In conclusion, we have systematically characterized the prevalence of the FN1-FGFR1 and the novel FN1-FGF1 fusions in a large group of wellcharacterized phosphaturic mesenchymal tumors. Our findings suggest the central role of FGFR1 signaling in the pathogenesis of phosphaturic mesenchymal tumors. Future work is required to elucidate the functional aspect of these fusion proteins, as well as the tumorigenic drivers behind fusion-negative cases.

\section{Acknowledgments}

This work was supported by research funds from Ministry of Science and Technology, Taiwan (MOST 104-2628-B-002-003-MY2) and National Taiwan University Hospital, Taipei, Taiwan (NTUH 105-S3041) to Dr Jen-Chieh Lee. We thank Dr Chih-Chien Hung for his assistance in collecting materials; Mr Yung-Chuan Chung, Mr Ren-Wei Wang, Ms Ying-Ying Chuang, and the NTUH sequencing core laboratory for the technical assistance; and Mr Grant Perry for his selfless dedications and generous contributions to this research. URLs for the raw data of RNA-seq: http://www.ncbi.nlm.nih.gov/sra/?term = SRX1560738 (sample PMT-50) http://www.ncbi.nlm.nih.gov/sra/? term = SRX1560739 (sample PMT-16 and -17).

\section{Disclosure/conflict of interest}

The authors declare no conflict of interest.

\section{References}

1 Folpe AL. Phosphaturic mesenchymal tumourIn: Fletcher CDM, Bridge JA, Hogendoorn PCW et al. eds World Health Organization Classification of Tumours of Soft Tissue and Bone. IARC Press: Lyon, France, 2013;2.

2 Folpe AL, Fanburg-Smith JC, Billings SD et al. Most osteomalacia-associated mesenchymal tumors are a single histopathologic entity: an analysis of 32 cases and a comprehensive review of the literature. Am J Surg Pathol 2004;28:1-30.

3 Shimada T, Mizutani S, Muto $\mathrm{T}$ et al. Cloning and characterization of FGF23 as a causative factor of tumor-induced osteomalacia. Proc Natl Acad Sci USA 2001;98:6500-6505.

4 White KE, Larsson TE, Econs MJ. The roles of specific genes implicated as circulating factors involved in normal and disordered phosphate homeostasis: frizzled related protein-4, matrix extracellular phosphoglycoprotein, and fibroblast growth factor 23. Endocr Rev 2006;27:221-241.

5 Berndt T, Craig TA, Bowe AE et al. Secreted frizzledrelated protein 4 is a potent tumor-derived phosphaturic agent. J Clin Invest 2003;112:785-794.

6 Rowe PS, de Zoysa PA, Dong R et al. MEPE, a new gene expressed in bone marrow and tumors causing osteomalacia. Genomics 2000;67:54-68.
7 Habra MA, Jimenez C, Huang SC et al. Expression analysis of fibroblast growth factor-23, matrix extracellular phosphoglycoprotein, secreted frizzled-related protein-4, and fibroblast growth factor-7: identification of fibroblast growth factor-23 and matrix extracellular phosphoglycoprotein as major factors involved in tumor-induced osteomalacia. Endocr Pract 2008;14: 1108-1114.

8 Lee JC, Jeng YM, Su SY et al. Identification of a novel FN1-FGFR1 genetic fusion as a frequent event in phosphaturic mesenchymal tumour. J Pathol 2015;235: 539-545.

9 Bowe AE, Finnegan R, Jan de Beur SM et al. FGF-23 inhibits renal tubular phosphate transport and is a PHEX substrate. Biochem Biophys Res Commun 2001;284:977-981.

10 Carter JM, Caron BL, Dogan et al. A novel chromogenic in situ hybridization assay for FGF23 mRNA in phosphaturic mesenchymal tumors. Am J Surg Pathol 2015; 39:75-83.

11 Trapnell C, Pachter L, Salzberg SL. TopHat: discovering splice junctions with RNA-Seq. Bioinformatics 2009;25:1105-1111.

12 Anders S, McCarthy DJ, Chen Y et al. Count-based differential expression analysis of RNA sequencing data using $\mathrm{R}$ and Bioconductor. Nat Protoc 2013;8: 1765-1786.

13 Lee JC, Lu TP, Changou CA et al. Genomewide copy number analysis of Müllerian adenosarcoma identified chromosomal instability in the aggressive subgroup. Mod Pathol; advance online publication, 3 June 2016; doi:10.1038/modpathol.2016.99 (e-pub ahead of print).

14 Weidner N, Santa Cruz D. Phosphaturic mesenchymal tumors. A polymorphous group causing osteomalacia or rickets. Cancer 1987;59:1442-1454.

15 Dorfman HD, Czerniak B. Bone Tumors. Mosby: St Louis, MO, USA, 1998.

16 Jackson CC, Medeiros LJ, Miranda RN. 8p11 myeloproliferative syndrome: a review. Hum Pathol 2010;41: 461-476.

17 Ren H, Tan ZP, Zhu X et al. Identification of anaplastic lymphoma kinase as a potential therapeutic target in ovarian cancer. Cancer Res 2012;72:3312-3323.

18 Lovly CM, Gupta A, Lipson D et al. Inflammatory myofibroblastic tumors harbor multiple potentially actionable kinase fusions. Cancer Discov 2014;4: 889-895.

19 el-Hariry I, Pignatelli M, Lemoine N. Fibroblast growth factor 1 and fibroblast growth factor 2 immunoreactivity in gastrointestinal tumours. J Pathol 1997;181:39-45.

20 Chow NH, Cheng KS, Lin PW et al. Expression of fibroblast growth factor-1 and fibroblast growth factor-2 in normal liver and hepatocellular carcinoma. Dig Dis Sci 1998;43:2261-2266.

21 Smith J, Yelland A, Baillie R et al. Acidic and basic fibroblast growth factors in human breast tissue. Eur J Cancer 1994;30A:496-503.

22 Kwabi-Addo B, Ozen M, Ittmann M. The role of fibroblast growth factors and their receptors in prostate cancer. Endocr Relat Cancer 2004;11:709-724.

23 Ornitz DM, Xu J, Colvin JS et al. Receptor specificity of the fibroblast growth factor family. J Biol Chem 1996;271:15292-15297.

24 Gospodarowicz D, Handley HH. Stimulation of division of Y1 adrenal cells by a growth factor isolated from bovine pituitary glands. Endocrinology 1975;97: 102-107. 
25 Beenken A, Mohammadi M. The FGF family: biology, pathophysiology and therapy. Nat Rev Drug Discov 2009;8:235-253.

26 Imamura T, Engleka K, Zhan X et al. Recovery of mitogenic activity of a growth factor mutant with a nuclear translocation sequence. Science 1990;249: 1567-1570.

27 Wesche J, Malecki J, Wiedlocha et al. Two nuclear localization signals required for transport from the cytosol to the nucleus of externally added FGF-1 translocated into cells. Biochemistry 2005;44:6071-6080.

28 Singh P, Carraher C, Schwarzbauer JE. Assembly of fibronectin extracellular matrix. Annu Rev Cell Dev Biol 2010;26:397-419.

29 Quade BJ, McDonald JA. Fibronectin's amino-terminal matrix assembly site is located within the $29-\mathrm{kDa}$ amino-terminal domain containing five type I repeats. J Biol Chem 1988;263:19602-19609.

$30 \mathrm{Wu}$ ZL, Zhang L, Yabe $\mathrm{T}$ et al. The involvement of heparan sulfate (HS) in FGF1/HS/FGFR1 signaling complex. J Biol Chem 2003;278:17121-17129.

31 Brown A, Robinson CJ, Gallagher JT et al. Cooperative heparin-mediated oligomerization of fibroblast growth factor-1 (FGF1) precedes recruitment of FGFR2 to ternary complexes. Biophys J 2013;104:1720-1730.

32 Nieto L, Canales A, Fernandez IS et al. Heparin modulates the mitogenic activity of fibroblast growth factor by inducing dimerization of its receptor. a 3D view by using NMR. Chembiochem 2013;14:1732-1744.

33 Puls F, Hofvander J, Magnusson L et al. FN1-EGF gene fusions are recurrent in calcifying aponeurotic fibroma. J Pathol 2016;238:502-507.

34 Jeon E, Kim HW, Jang JH. Protein engineering of a fibroblast growth factor-1 fusion protein with cell adhesive activity. Acta Biochim Biophys Sin (Shanghai) 2009;41:852-857.

35 Brownstein CA, Adler F, Nelson-Williams C et al. A translocation causing increased alpha-klotho level results in hypophosphatemic rickets and hyperparathyroidism. Proc Natl Acad Sci USA 2008;105:3455-3460.
36 Nabeshima Y. Klotho: a fundamental regulator of aging. Ageing Res Rev 2002;1:627-638.

37 White KE, Cabral JM, Davis SI et al. Mutations that cause osteoglophonic dysplasia define novel roles for FGFR1 in bone elongation. Am J Hum Genet 2005;76: 361-367.

38 Liu S, Tang W, Fang J et al. Novel regulators of Fgf23 expression and mineralization in Hyp bone. Mol Endocrinol 2009;23:1505-1518.

39 Martin A, Liu S, David V et al. Bone proteins PHEX and DMP1 regulate fibroblastic growth factor Fgf23 expression in osteocytes through a common pathway involving FGF receptor (FGFR) signaling. FASEB J 2011;25: 2551-2562.

$40 \mathrm{Wu}$ AL, Feng B, Chen MZ et al. Antibody-mediated activation of FGFR1 induces FGF23 production and hypophosphatemia. PLoS One 2013;8:e57322.

41 Xiao Z, Huang J, Cao L et al. Osteocyte-specific deletion of Fgfr1 suppresses FGF23. PLoS One 2014;9:e104154.

42 Yavropoulou MP, Gerothanasi N, Frydas et al. Tumorinduced osteomalacia due to a recurrent mesenchymal tumor overexpressing several growth factor receptors. Endocrinol Diabetes Metab Case Rep 2015;2015: 150025.

43 Bahrami A, Weiss SW, Montgomery E et al. RT-PCR analysis for FGF23 using paraffin sections in the diagnosis of phosphaturic mesenchymal tumors with and without known tumor induced osteomalacia. Am J Surg Pathol 2009;33:1348-1354.

44 Doyle LA, Vivero M, Fletcher CD et al. Nuclear expression of STAT6 distinguishes solitary fibrous tumor from histologic mimics. Mod Pathol 2014;27: 390-395.

45 Behjati S, Tarpey PS, Presneau N et al. Distinct H3F3A and H3F3B driver mutations define chondroblastoma and giant cell tumor of bone. Nat Genet 2013;45: 1479-1482.

46 Rosenbloom KR, Armstrong J, Barber GP et al. The UCSC Genome Browser database: 2015 update. Nucleic Acids Res 2015;43:D670-D681.

Supplementary Information accompanies the paper on Modern Pathology website (http://www.nature.com/ modpathol) 